\title{
Diagnostic Sensitivity of Different Applications of Pathergy Test for Behçet's Disease
}

\author{
İncilay KALAY YILDIZHAN 1 , Ayse BOYVAT (1) \\ Department of Dermatology, Ankara University School of Medicine, Ankara, Turkey
}

\begin{abstract}
Objectives: This study aims to compare the sensitivity of the intradermal application of the pathergy test with the "three-step pathergy test". Patients and methods: The study included 60 patients with Behçet's disease (BD) (23 males, 37 females; mean age $33.9 \pm 9.7$ years; range, 11 to 57 years) and 100 patients with recurrent aphthous stomatitis as the control group (39 males, 61 females; mean age $32.4 \pm 12.1$ years; range, 11 to 66 years). Simultaneous intradermal pathergy test and three-step pathergy test were applied to each patient.

Results: Twenty-six patients (43.3\%) with BD had positive three-step pathergy test and 18 (30\%) of them had positive intradermal pathergy test. A statistically significant relationship was found between the three-step pathergy test positivity and male sex while no relationship was detected between the disease activity and the positivity of the pathergy test. In the three-step pathergy test, pathergy positivity with intramuscular method was statistically significantly higher than with intravenous method.

Conclusion: The three-step pathergy test in patients with BD was found to have higher sensitivity compared with the intradermal pathergy test. The intramuscularly applied pathergy test was the main cause of increase in sensitivity. Further studies are needed to develop new applications of pathergy test that would increase the sensitivity and which are also easy to apply.

Keywords: Behçet's disease, diagnosis, disease activity, pathergy test, sensitivity.
\end{abstract}

Behçet's disease (BD) is a chronic inflammatory disease that presents with mucocutaneous findings and ocular, vascular, articular, neurological and gastrointestinal tract involvement. There is no specific laboratory test for the diagnosis of $\mathrm{BD}$, and the diagnosis can only be established with sufficient medical history and evaluation of the clinical findings. The pathergy reaction, first described by Blobner in 1937, manifests itself by the development of an erythematous papule or pustule at the injection site 24-48 hours after an intradermal puncture. ${ }^{1}$ The pathergy test is used as an adjunctive test in the diagnosis of $\mathrm{BD}$ and indicates hyperreactivity of the skin. Currently, there is no standardization of the application of the pathergy test and the most commonly used method is the intradermal application of a 20-22 gauge needle at an angle of 45 degrees to at least two different sites on an avascular area on the forearm. Erdem et al. ${ }^{2}$ have conducted a study at our department in 1987 about a new application method for the pathergy test, which is known as the "three-step pathergy test". Taking into consideration the possibility that pathergy test reactions may differ at different times, the pathergy test was applied as intramuscular and intravenous punctures on three consecutive days and the puncture sites were evaluated at $24^{\text {th }}$ and $48^{\text {th }}$ hours. Pathergy positivity was detected among $74 \%$ of the patients with this method.

Received: January 08, 2019 Accepted: March 01, 2019 Published online: April 15, 2019

Correspondence: İncilay Kalay Yıldızhan, MD. Ankara Üniversitesi Tıp Fakültesi Deri ve Zührevi Hastalıkları Anabilim Dalı, 06100 Tandoğan, Ankara. Tel: +90 505 - 6547721 e-mail: incilaykalay@gmail.com 
Since the prevalence of the positivity of the pathergy test has declined in the recent years, studies have been performed to increase the specificity, sensitivity and reliability of the test. ${ }^{2}$ In this study, we aimed to compare the sensitivity of the intradermal application of the pathergy test with the "three-step pathergy test".

\section{PATIENTS AND METHODS}

The study was conducted at Ankara University School of Medicine between January 2013 and January 2014 and included 60 patients (23 males, 37 females; mean age 33.9 9 9.7 years; range, 11 to 57 years) who met the diagnosis of $\mathrm{BD}$ regardless of pathergy positivity ${ }^{3,4}$ and 100 patients with recurrent aphthous stomatitis as the control group (39 males, 61 females; mean age $32.4 \pm 12.1$ years; range, 11 to 66 years). Newly diagnosed BD patients and patients who had discontinued the therapy for various reasons were included. Patients using colchicine, antiinflammatory drugs and immunosuppressive therapies, such as corticosteroids, cyclosporine, and azathioprine were excluded because of their possible effect on pathergy reaction. Patients who had at least one of the following findings at the time of pathergy test application were considered to have active disease: genital ulcer, erythema nodosum-like skin lesion in addition to oral aphthous ulcers and patients with active uveitis, joint involvement, vascular, gastrointestinal or neurological involvement. Three-step pathergy test and intradermal pathergy test were applied simultaneously to each patient. The application sites were cleaned with 70\% isopropyl alcohol before each puncture and 20 gauge syringe needle (Ayset Medical Products, Adana, Turkey) was used. The study protocol was approved by the Ankara University School of Medicine Ethics Committee (approval number: 20-651-12). A written informed consent was obtained from each patient. The study was conducted in accordance with the principles of the Declaration of Helsinki.

Intradermal pathergy test was applied as three punctures to the volar aspect of the forearm on the first day. Intradermal pathergy test was considered as positive when a positive reaction was detected in at least one of the three punctures at 24 or 48 hours. The application and evaluation of the intradermal test therefore took three days.

In three-step pathergy test, intravenous puncture was applied to the antecubital vein and $1 \mathrm{~mL}$ saline was injected intramuscularly to upper external quadrant of the glutea on three consecutive days. The puncture sites were evaluated at 24 and 48 hours and pathergy test was considered as positive in the presence of a positive reaction in at least one of the intravenous or intramuscular pathergy sites. The application and evaluation period of the three-step pathergy test was therefore longer and took five days.

\section{Statistical analysis}

Statistical analysis was performed using the IBM SPSS version 21.0 software (IBM Corp., Armonk, NY, USA). The data were summarized by frequencies, percentages, arithmetic means and standard deviation. McNemar, t-test, Chi square and Mann-Whitney $U$ tests were used to compare the groups. A $p$ value of $<0.05$ was considered statistically significant.

\section{RESULTS}

In our study, no difference was determined among the patient and control groups in terms of age and sex ( $>0.05)$. All of our BD patients had oral aphthae (100\%), 48 patients had genital ulcers (80\%), 48 patients had papulopustular skin lesions (80\%), 34 patients had arthralgia (56.7\%), 26 patients had erythema nodosum-like skin lesions (43.3\%), nine patients had ocular involvement (15\%), two patients had vascular involvement (3.3\%) and one patient had gastrointestinal involvement (1.7\%). Twenty one $\mathrm{BD}$ patients (35\%) were in the active stage and 39 patients (65\%) were in remission. Genital ulcers were present in 19 of the active patients followed by erythema nodosum in four patients and uveitis in one patient.

Twenty-seven patients (45\%) with BD had positive three-step pathergy test and/or intradermal pathergy test (Figure 1). Twenty-six (43.3\%) of them had positive three-step pathergy test and 18 (30\%) had positive intradermal pathergy test. Seventeen patients (28.3\%) had pathergy test positivity with both of the intradermal and three-step pathergy tests. One patient (1.7\%) had only intradermal 

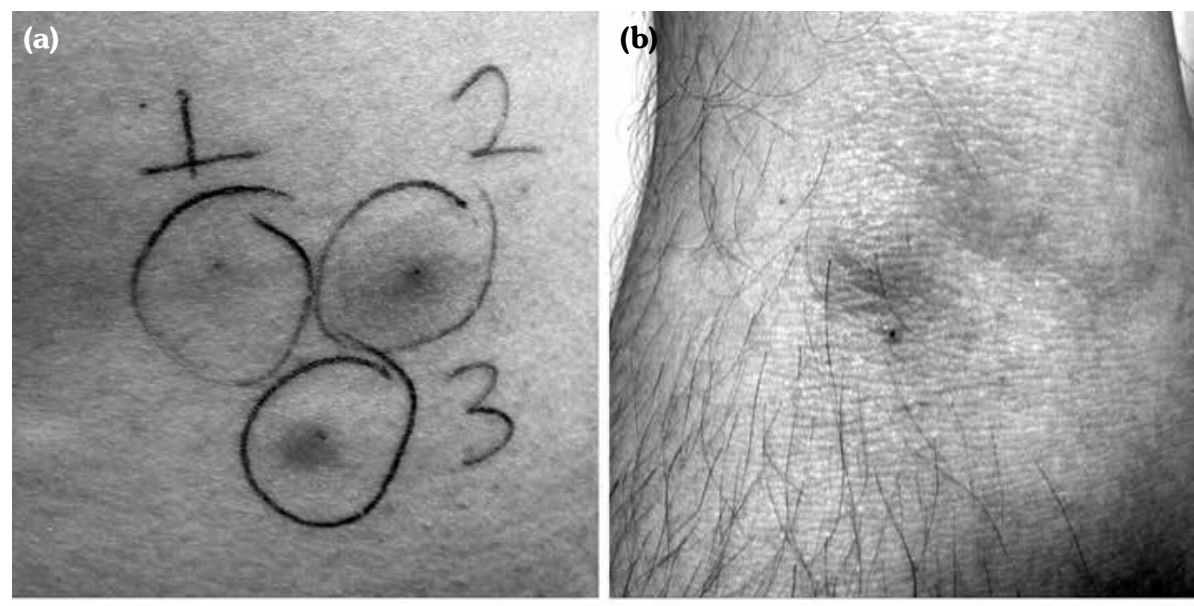

(c)

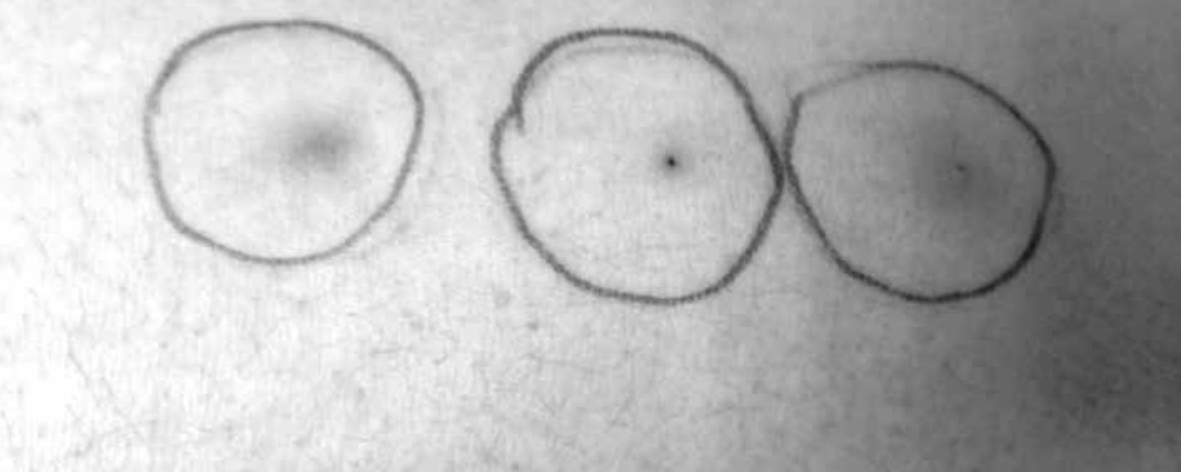

Figure 1. (a) Positive pathergy reaction at intramuscular needle prick sites with threestep pathergy test applied on three consecutive days. (b) Positive patch test reaction at intravenous needle prick site with three-step pathergy test. (c) Positive patch test reactions at intradermal pathergy sites.

pathergy test positivity and nine patients (15\%) had only three-step pathergy test positivity. Pathergy test was negative in 33 patients with $\mathrm{BD}$. In the control group, four patients (4\%) had three-step pathergy test positivity and two patients (2\%) had intradermal pathergy test positivity. The sensitivity and specificity of the three-step pathergy test were $43 \%$ and $96 \%$, respectively. The intradermal pathergy test had a sensitivity of $30 \%$ and a specificity of $98 \%$. The sensitivity of three-step pathergy test was significantly higher than the sensitivity of the intradermal pathergy test $(p<0.05)$.

A statistically significant relationship was found between the positivity of the three-step pathergy test and male sex $(p<0.05)$. Both the intradermal pathergy test and the three-step pathergy test were found to be more frequently positive in patients under 40 years of age, but the difference was not statistically significant ( $p>0.05$ ). There was no statistically significant relationship between disease activity and the positivity of the intradermal test or the three-step pathergy test ( $p>0.05)$.

In all of the intradermal, intramuscular and intravenous pathergy tests, increase in the number of punctures increased the rate of the pathergy positivity. Compared with a single puncture application, application of three punctures increased intradermal pathergy test positivity from 21 to $30 \%$, intramuscular test positivity from 30 to $43.3 \%$, and intravenous test positivity from 10 to $21.6 \%$. 


\section{DISCUSSION}

Significant ethnic differences are observed in the rates of the pathergy positivity in BD. While the rate of pathergy test positivity is higher in Japan, Turkey and Eastern Mediterranean countries with a range of $50-88 \%$, the positivity rates in England and USA are below 20\% and the diagnostic significance of the test is therefore quite low in these countries. ${ }^{5-11}$ Recent studies have revealed a decrease in the rate of pathergy positivity. In different studies conducted in Turkey in the last 10 years, the rate of the pathergy positivity ranged from 40 to $45.3 \% .^{12-14}$ In our study, the pathergy test was found to be positive among $45 \%$ of the patients.

Pathergy positivity may be affected by factors such as age and sex of the patient and disease activity. In some studies, pathergy positivity was found to be more frequent in male patients. ${ }^{15,16}$ Yazıc1 et al. ${ }^{17}$ have reported similar pathergy positivity rates in male and female patients; however, reaction was noted to be more severe in male patients. In our study, the positivity of both the intradermal test and the three-step pathergy test was found to be higher in male patients; however, statistically significant difference was only found in the three-step pathergy test $(p<0.05)$. Jorizzo et al. $^{18}$ and Akmaz et al. ${ }^{19}$ reported higher rates of pathergy positivity among patients in the active stage, compared with patients in remission. On the other hand, some researchers suggested that the pathergy reaction is not related to the disease activity. ${ }^{17,20}$ In our study, there was no statistically significant relationship between disease activity and the positivity of the three-step pathergy test and the intradermal pathergy test ( $p>0.05)$.

Today, an important problem is the lack of standardization in the application of the pathergy test. Factors such as needle thickness, sharpness or bluntness of the needle tip, number of punctures, and whether or not the test area has been cleaned with an antiseptic have all been shown to affect the positivity rate of the test. ${ }^{19,21-25}$ In a study carried out with 270 dermatologists in Turkey, different application methods of pathergy test were investigated and according to the results of this study, $76.1 \%$ of physicians used the intradermal method, all of them preferred forearm for the test, $46.9 \%$ applied three punctures, most of them abandoned using blunt-tipped needles and $69.7 \%$ cleaned the test area with alcohol. ${ }^{26}$

According to the results of our study, the sensitivity of the three-step pathergy test was higher than the intradermal pathergy test. The higher rate of the positivity of the three-step pathergy test may be attributed to the increased number of punctures. However, in the threestep pathergy test, higher frequency of positivity was associated primarily with the intramuscular method, and the intravenous method did not contribute to the positivity of the test. The rates of pathergy test positivity following three intradermal, intramuscular and intravenous punctures were $30 \%, 43.3 \%$, and $21.6 \%$, respectively.

In our study, saline injection was also applied in the intramuscular method. Therefore, it can be argued that high positivity by intramuscular method may be related to saline injection. However, Askari et al. ${ }^{27}$ have found no effects of saline injection on the rate of pathergy positivity. We therefore think that the relationship between saline injection and the higher rate of pathergy positivity should be investigated in further studies.

The intravenous application did not contribute to the rate of the positivity of three-step pathergy test. We can therefore conclude that the intravenous pathergy test should not be preferred over the other methods because of the difficulty in application and the lower rate of positivity. We think that intramuscular and intradermal pathergy tests are more suitable methods due to their easier applicability and higher rate of positivity.

Özdemir et al. ${ }^{24}$ studied pathergy test positivity on different body parts, such as forearm, leg, back and abdomen. The authors found the highest rate of positivity on the forearm, and the lowest rate of positivity on the abdominal region. The authors attributed these differences to the differences in the thickness and vascular structures of the skin on different body parts. In our study, intramuscular injections were applied to the gluteal region. It would be appropriate to investigate the positivity of the intramuscular pathergy test on different body regions in further studies.

In the intradermal pathergy test, the patients are assessed at $24^{\text {th }}$ and $48^{\text {th }}$ hours and the test is completed within three days. On the other hand, 
three-step pathergy test is applied over three consecutive days and the test takes five days. While this may increase the sensitivity of the pathergy test, the necessity to visit the hospital for five days may compromise patient compliance and impose application difficulties.

The fact that our study was designed to compare the sensitivity of the intradermal pathergy test with the sensitivity of the three-step pathergy test and therefore it did not allow direct comparison of intramuscular, intravenous and intradermal administration due to methodological differences may be considered a limitation. However, the findings of our study may inspire new studies on the application of pathergy test.

In conclusion, our study showed that the three-step pathergy test was more sensitive than the intradermal pathergy test. In the three-step pathergy test, higher frequency of positivity was associated primarily with the intramuscular method, and the intravenous method did not contribute to the positivity of the test. In both the intradermal pathergy test and the three-step pathergy test, increased number of punctures was found to increase the rate of positivity. For this reason, while planning the pathergy test, it is appropriate to prefer intradermal and intramuscular routes with application of multiple punctures. Well-constructed studies are needed to compare the sensitivity of intradermal and intramuscular pathergy tests.

\section{Declaration of conflicting interests}

The authors declared no conflicts of interest with respect to the authorship and/or publication of this article.

\section{Funding}

The authors received no financial support for the research and/or authorship of this article.

\section{REFERENCES}

1. Blobner F. Zur rezidivierenden Hypopyoniritis. Z Augenheilkd 1937;91:129-39.

2. Erdem C, Gürler A, Kundakçı N. Behçet hastalarında üç aşamalı paterji testi ile elde edilen sonuçlar. Lepr Mec 1987;18:73-84.

3. Criteria for diagnosis of Behçet's disease. International Study Group for Behçet's Disease. Lancet 1990;335:1078-80.
4. International Team for the Revision of the International Criteria for Behcet's Disease. Evaluation of the International Criteria for Behcet's disease (ICBD) Clinical and Experimental Rheumatology 2006;24(Suppl 42):S13.

5. Gürler A, Boyvat A, Türsen U. Clinical manifestations of Behçet's disease: an analysis of 2147 patients. Yonsei Med J 1997;38:423-7.

6. Dogan B, Taskapan O, Harmanyeri Y. Prevalance of pathergy test positivity in Behçet's disease in Turkey. J Eur Acad Dermatol Venereol 2003;17:228-9.

7. Lee ES, Bang D, Lee S. Dermatologic manifestation of Behçet's disease. Yonsei Med J 1997;38:380-9.

8. Yazici $H$, Chamberlain MA, Tüzün Y, Yurdakul $S$, Müftüoglu A. A comparative study of the pathergy reaction among Turkish and British patients with Behçet's disease. Ann Rheum Dis 1984;43:74-5.

9. Davies PG, Fordham JN, Kirwan JR, Barnes CG, Dinning WJ. The pathergy test and Behçet's syndrome in Britain. Ann Rheum Dis 1984;43:70-3.

10. Tüzün $Y$, Yurdakul $S$, Cem Mat M, Ozyazgan $Y$, Hamuryudan V, Tüzün $B$, et al. Epidemiology of Behçet's syndrome in Turkey. Int $\mathrm{J}$ Dermatol 1996;35:618-20.

11. International Team for the Revision of the International Criteria for Behçet's Disease (ITRICBD). The International Criteria for Behçet's Disease (ICBD): a collaborative study of 27 countries on the sensitivity and specificity of the new criteria. J Eur Acad Dermatol Venereol 2014;28:338-47.

12. Atalay A, Arıkan S, Ozturk O, Öncü M, Tasli ML, Duygulu S, et al. The IL-8 Gene Polymorphisms in Behçet's Disease Observed in Denizli Province of Turkey. Immunol Invest 2016;45:298-311.

13. Aydin F, Akpolat T, Senturk N, Bagci H, Yasar Turanli A. Evaluation of pathergy test positivity in familial Mediterranean fever patients and comparison of clinical manifestations of FMF with Behçet's disease. Clin Rheumatol 2009;28:1331-5.

14. Balta I, Akbay G, Kalkan G, Eksioglu M. Demographic and clinical features of 521 Turkish patients with Behçet's disease. Int J Dermatol 2014;53:564-9.

15. Alli N, Gur G, Yalcin B, Hayran M. Patient characteristics in Behçet disease: a retrospective analysis of 213 Turkish patients during 2001-4. Am J Clin Dermatol 2009;10:411-8.

16. Saylan T, Mat C, Fresko I, Melikoğlu M. Behçet's disease in the Middle East. Clin Dermatol 1999;17:209-23.

17. Yazici $H$, Tüzün $Y$, Tanman $A B$, Yurdakul $S$, Serdaroglu S, Pazarli $\mathrm{H}$, et al. Male patients with Behçet's syndrome have stronger pathergy reactions. Clin Exp Rheumatol 1985;3:137-41.

18. Jorizzo JL, Solomon AR, Cavallo T. Behcet's syndrome. Immunopathologic and histopathologic assessment of pathergy lesions is useful in diagnosis and follow-up. Archives of pathology \& laboratory medicine. 1985;109:747-51. 
19. Akmaz O, Erel A, Gürer MA. Comparison of histopathologic and clinical evaluations of pathergy test in Behçet's disease. Int $\mathrm{J}$ Dermatol 2000;39:121-5.

20. Krause I, Molad Y, Mitrani M, Weinberger A. Pathergy reaction in Behçet's disease: lack of correlation with mucocutaneous manifestations and systemic disease expression. Clin Exp Rheumatol 2000;18:71-4.

21. Ozarmagan G, Saylan T, Azizlerli G, Ovül C, Aksungur $\mathrm{VL}$. Re-evaluation of the pathergy test in Behçet's disease. Acta Derm Venereol 1991;71:75-6.

22. Dilşen N, Koniçe M, Aral O, Ocal L, Inanç M, Gül A. Comparative study of the skin pathergy test with blunt and sharp needles in Behçet's disease: confirmed specificity but decreased sensitivity with sharp needles. Ann Rheum Dis 1993;52:823-5.
23. Fresko I, Yazici H, Bayramiçli M, Yurdakul S, Mat C. Effect of surgical cleaning of the skin on the pathergy phenomenon in Behçet's syndrome. Ann Rheum Dis 1993;52:619-20.

24. Ozdemir M, Balevi S, Deniz F, Mevlitoğlu I. Pathergy reaction in different body areas in Behçet's disease. Clin Exp Dermatol 2007;32:85-7.

25. Ozdemir M, Bodur S, Engin B, Baysal I. Evaluation of application of multiple needle pricks on the pathergy reaction. Int J Dermatol 2008;47:335-8.

26. Ozden MG, Bek Y, Aydin F, Senturk N, Canturk T, Turanli AY. Different application techniques of pathergy testing among dermatologists. J Eur Acad Dermatol Venereol 2010;24:1240-2.

27. Askari A, Al-Aboosi M, Sawalha A. Evaluation of pathergy test in North Jordan. Clin Rheumatol 2000;19:249-51. 